\title{
KINETIC SIMULATIONS OF TURBULENT MAGNETIC-FIELD GROWTH BY STREAMING COSMIC RAYS
}

\author{
Thomas Stroman ${ }^{1}$, Martin PohL $^{1,3}$, and Jacek Niemiec ${ }^{2}$ \\ ${ }^{1}$ Department of Physics and Astronomy, Iowa State University, Ames, IA 50011, USA \\ ${ }^{2}$ Institute of Nuclear Physics PAN, ul. Radzikowskiego 152, 31-342 Kraków, Poland \\ Received 2009 August 31; accepted 2009 September 30; published 2009 October 27
}

\begin{abstract}
Efficient acceleration of cosmic rays (via the mechanism of diffusive shock acceleration) requires turbulent, amplified magnetic fields in the shock's upstream region. We present results of multidimensional particle-in-cell simulations aimed at observing the magnetic field amplification that is expected to arise from the cosmic-ray current ahead of the shock, and the impact on the properties of the upstream interstellar medium. We find that the initial structure and peak strength of the amplified field are somewhat sensitive to the choice of parameters, but that the field growth saturates in a similar manner in all cases: the back-reaction on the cosmic rays leads to modification of their rest-frame distribution and also a net transfer of momentum to the interstellar medium, substantially weakening their relative drift while also implying the development of a modified shock. The upstream medium becomes turbulent, with significant spatial fluctuations in density and velocity, the latter in particular leading to moderate upstream heating; such fluctuations will also have a strong influence on the shock structure.
\end{abstract}

Key words: acceleration of particles - cosmic rays - methods: numerical - shock waves - supernova remnants turbulence

Online-only material: color figures

\section{INTRODUCTION}

The forward shocks of young shell-type supernova remnants may be efficient acceleration sites for Galactic cosmic rays. The theory of diffusive shock acceleration (DSA) provides a promising mechanism by which a small fraction of particles can attain nonthermal energies in such an environment, provided they are confined to the shock vicinity; the review by Reynolds (2008) and its references have treated the subject in detail. This confinement may be supplied to some degree by the interstellar magnetic field, but the estimated upper limit in energy falls short of what is observed in cosmic rays of Galactic origin. The implied need for a much stronger, turbulent upstream field, together with observational evidence for strong magnetic fields immediately behind the forward shock, has attracted attention to the question of upstream magnetic-field amplification.

It is well known that ion beams can generate magnetic turbulence via resonant and non-resonant interactions (Winske and Leroy 1984). Bell (2004, hereafter B04) suggested that nonresonant magnetic field amplification may also be caused by the cosmic-ray current expected in the cosmic-ray precursor of a quasi-parallel shock. Although substantial field amplification has occurred in magnetohydrodynamical (MHD) simulations that assume a constant cosmic-ray current (B04; also Zirakashvili et al. 2008), the approximations of MHD may not be appropriate for modeling the nonlinear evolution and eventual saturation of this instability; we must use kinetic methods to simulate these later stages with accuracy, including any backreaction on the cosmic rays.

Our earlier work (Niemiec et al. 2008, hereafter N08) used two-dimensional and three-dimensional particle-in-cell (PIC) simulations to model the growth and saturation of a currentdriven instability. We relaxed the condition assumed in the calculations of B04 that the rate of unstable growth must be much less than the ion gyrofrequency, and we found that an

\footnotetext{
3 Now at Institut für Physik und Astronomie, Universität Potsdam, 14476 Potsdam-Golm, Germany and DESY, 15738 Zeuthen, Germany
}

oblique filamentary mode dominated the initial magnetic-field growth. Riquelme and Spitkovsky (2009) confirmed the findings of N08, and in particular investigated the parameters for the transition between the filamentation mode seen in N08 and the parallel-wave mode seen in the MHD simulations, and verified that the parallel mode appears only in the regime with $\omega \ll \Omega_{i}$. However, despite the difference in initial unstable modes, the nonlinear characteristics of the system observed in both works (N08; Riquelme and Spitkovsky 2009) were similar. In particular, both investigations observed only modest amplification of the magnetic field, and saturation by the same mechanism. The analytical calculations of Luo and Melrose (2009), with a kinetic treatment of the current-driven instability, predict a saturation level consistent with the kinetic simulations.

In this paper, we report more recent work, in which we simulate the current-driven instability and its saturation in a parameter regime in which the non-relativistic ion gyrofrequency far exceeds the (complex) frequency of the current-driven instability. Such parameters are particularly relevant to the environment of non-relativistic strong shocks whose cosmic-ray precursors are sparse in comparison with the magnetized ambient interstellar medium (ISM); an understanding of the limits of field amplification in such an environment is therefore essential to the formulation of a self-consistent theory of cosmic-ray acceleration in the Galaxy. We find that the initial evolution of the magnetic field in this parameter regime is in agreement with the predictions of B04 and consistent with the transition parameter, $\omega / \Omega_{i}$, identified by Riquelme and Spitkovsky (2009). We observe that the field growth is limited to modest values by the same saturation mechanism as before (N08; Riquelme and Spitkovsky 2009). In addition to the magnetic field, we examine the evolution of the upstream medium-precursor system in terms of bulk and local properties that are relevant for modeling the shock acceleration process. Notable findings include the rise of strong turbulent motion relative to the bulk upstream rest frame, and of motional electric fields associated with the magnetic fields that permeate the turbulent flow. The temperature of the upstream medium, measured in the local rest frame as determined 
with high spatial resolution, attains significant values during the evolutionary phase characterized by the turbulent motion, such that the ratio of magnetic to thermal energy density may in fact decrease as a result of this precursor-ISM interaction. Such modifications to the upstream medium may lead to substantial effects on the propagation of the subshock, and on the properties of the downstream medium: both the pre-existing upstream turbulence after advection and shock compression, and further magnetic-field generation via turbulent dynamo processes, may be quite sensitive to turbulence in the upstream medium (Giacalone and Jokipii 2007; Inoue et al. 2009).

We begin by describing in Section 2.1 the simple physical scenario that captures all the ingredients necessary for the streaming instability we want to simulate, with Section 2.2 summarizing our computational representation of that environment, and the specific parameterizations being outlined in Section 2.3. We present our simulation results in Section 3 along with a discussion of their significance, and finally summarize in Section 4.

\section{SIMULATION SETUP}

\subsection{Model}

To study the growth and saturation of magnetic turbulence in response to a cosmic-ray current, we simulate the evolution of a system representative of the upstream environment of a non-relativistic strong parallel shock. We assume that the interstellar medium is a homogeneous collisionless plasma composed entirely of ions and electrons (number density $N_{i}$ and $N_{e}$, respectively) of opposite but equal charge $\pm e$, permeated with a uniform magnetic field $B_{\| 0}$. A population of cosmic-ray ions (density $N_{\mathrm{CR}}=N_{e}-N_{i}$ ) drifts along the magnetic field at the shock velocity $v_{\mathrm{sh}}$ relative to the plasma and carries a current density $j_{\mathrm{CR}}=e N_{\mathrm{CR}} v_{\mathrm{sh}}$; the slight electron excess in the plasma maintains charge neutrality, and the electrons drift relative to the ions with $v_{d}=v_{\mathrm{sh}} N_{\mathrm{CR}} / N_{e}$ to provide a return current density $j_{r e t}=-e N_{e} v_{d}=-j_{\mathrm{CR}}$. The ISM ion and electron populations are each distributed according to Maxwell-Boltzmann statistics in their respective rest frames with the same temperature, given by the electron thermal velocity $v_{e, \text { th }}$.

Two assumptions in our setup, that only electrons provide the return current and that the streaming cosmic rays consist only of ions, arise from the large mass ratio $m_{i} / m_{e}$ between physical ions and electrons. The electrons will respond to any local charge or current imbalance on a much shorter timescale than the ions will. Moreover, at high energies, ions outnumber electrons (on account of their greater rigidity) by a factor that depends on the spectral index of their momentum distribution but is generally large enough that cosmic-ray electrons play a negligible role in the precursor physics. Cosmic rays upstream undergo pitch-angle scattering off magnetic inhomogeneities and approach a quasi-isotropic distribution, forming a steady cosmic-ray precursor in the shock rest frame. Ions with lower energies will be advected back toward the shock sooner than ions with higher energies, so at some distance upstream one expects to find a population of high-energy cosmic-ray ions drifting with the shock, up to some cutoff energy and/or distance (Reville et al. 2009) associated with particles that escape the system.

For our simulation, we simplify the cosmic-ray distribution function. We assume that the isotropic, scattered cosmic rays of the precursor are energetically much more significant than any escaping cosmic rays that happen to be passing through the region of our simulation, so we neglect the latter altogether. Our box size is assumed to be small in comparison both to the scale length of the cosmic-ray precursor, such that we may neglect spatial variations in their distribution, and to the Larmor radii of any cosmic rays present, such that the details of their energy distribution are similarly negligible, and we replace the already narrow range of energies with a single energy (in the CR population rest frame), with Lorentz factor $\gamma_{\mathrm{CR}}$. The quasi-linear calculations in B04 do not depend explicitly on cosmic-ray energy, and tests with various values of $\gamma_{\mathrm{CR}}$ have not produced significantly different outcomes, so it is unlikely that our choice of energy distribution obscures behavior that a more realistic model would illuminate.

In considering the current carried by cosmic rays through a cold ambient plasma parallel to a uniform magnetic field, the calculations in B04 predict an instability in which the dominant mode, circularly polarized magnetic fluctuations with wavenumber $k_{\| \max }$, grows at a rate $\operatorname{Im} \omega=\gamma_{\max }=v_{A} k_{\| \max }$, $\operatorname{Re} \omega \approx 0$, where

$$
k_{\| \max }=\frac{e N_{\mathrm{CR}} v_{\mathrm{sh}} B_{\| 0}}{2 N_{i} m_{i} v_{A}^{2}} \approx \frac{\mu_{0} J_{\mathrm{CR}}}{2 B_{\| 0}}
$$

and $v_{A}=\left[B_{\| 0}^{2} / \mu_{0}\left(N_{e} m_{e}+N_{i} m_{i}\right)\right]^{1 / 2}$ is the Alfvén velocity of the plasma. As mentioned above, this result is subject to the restriction that the fluctuation satisfy $\omega \ll \Omega_{i}$, that is, the growth rate of the instability must be much less than the nonrelativistic ion gyrofrequency.

\subsection{Implementation}

Our simulations employ a modified version of the particle-incell code TRISTAN (Buneman 1993). The code is updated to work in a high-performance computing environment and uses the charge-conserving current deposition algorithm of Umeda et al. (2003). We have incorporated a fourth-order-accurate algorithm for updating the electromagnetic fields based on Maxwell's equations (Greenwood et al. 2004), and adapted the code to a truly 2.5 -dimensional simulation. ${ }^{4}$

The shortest relevant physical time and lengthscales are given by the electron plasma period $\omega_{p e}^{-1}=\left[m_{e} \epsilon_{0} / e^{2} N_{e}\right]^{1 / 2}$ and electron skin depth $\lambda_{s e}=c / \omega_{p e}$, respectively. To ensure that physical small-scale electrostatic effects are distinct from those arising from the artificial discretization of space and time, for our simulations we choose parameters such that $\lambda_{s e} \geqslant 4 \Delta$, where $\Delta$ is the length of one cell, and $\omega_{p e}^{-1} \geqslant 8.9 \delta t$, where $\delta t$ is the timestep size. The wavelength $\lambda_{\max } \equiv 2 \pi / k_{\| \max }$ of the predicted magnetic-field fluctuations is of order $10^{2} \lambda_{s e}$ for all simulations (see Table 1). We perform our simulations in a rectangular computational grid, with both the homogeneous magnetic field and the direction of cosmic-ray drift aligned with the longer dimension. Periodic boundary conditions in both directions allow particles and fields to "wrap around" to the far side of the grid, but this also imposes an upper limit to the size of structures that can be represented accurately. Having observed a migration of the dominant structures toward increasing size scales in N08, for one simulation (Run A) we chose a grid slightly larger than $20 \lambda_{\max }$ in the drift direction, and $5 \lambda_{\max }$ in the transverse direction. We ran additional simulations on smaller grids to explore the influence of variations in our choice of parameters on the initial amplification of the magnetic field and the turbulent properties of the field and plasma.

\footnotetext{
4 As reported in N08, we have previously approximated two dimensions by simulating a volume with a thickness of only three grid cells.
} 
Table 1

Parameters and Selected Results of Simulations.

\begin{tabular}{lccccccccc}
\hline \hline Run & Grid $\left(\Delta^{2}\right)$ & $N_{i}\left(\Delta^{-2}\right)$ & $\lambda_{s e}(\Delta)$ & $v_{\text {sh }} / c$ & $\lambda_{\max }\left(\lambda_{s e}\right)$ & $\gamma_{\mathrm{CR}}$ & $B_{\perp} / B_{\| 0}$ & $\gamma / \gamma_{\max }$ & $\gamma_{\max } / \Omega_{i}$ \\
\hline A & $9000 \times 2400$ & 25 & 4 & 0.4 & 111 & 50 & 13.3 & 0.96 & 0.4 \\
$\mathrm{~A}^{\prime}$ & $3000 \times 1600$ & 25 & 4 & 0.4 & 111 & 50 & 17.6 & 0.86 & 0.4 \\
B & $4500 \times 1800$ & 20 & 6 & 0.4 & 111 & 50 & 19.7 & 0.89 & 0.4 \\
C & $4000 \times 1500$ & 25 & 5 & 0.3 & 148 & 10 & 11.6 & 0.78 & 0.3 \\
C $^{\prime}$ & $4000 \times 1500$ & 25 & 5 & 0.3 & 148 & 10 & 9.9 & 0.93 & 0.3 \\
D & $3200 \times 1200$ & 25 & 5 & 0.4 & 111 & 10 & 12.7 & 0.86 & 0.4 \\
E & $3200 \times 1200$ & 25 & 5 & 0.4 & 111 & 25 & 18.4 & 0.90 & 0.4 \\
F & $3200 \times 1200$ & 25 & 5 & 0.4 & 111 & 200 & 20.1 & 0.91 & 0.4 \\
\hline
\end{tabular}

Notes. Run $\mathrm{A}^{\prime}$ reproduces the parameters of Run A on a spatial scale comparable to run B. Run $\mathrm{C}^{\prime}$ differs from Run $\mathrm{C}$ only in that the cosmic-ray particles are not split into multiple simulated particles. $N_{i}$ is the number of ions per cell ( $\left.\operatorname{size} \Delta^{2}\right), \lambda_{s e}=c / \omega_{p e}$ is the electron skin depth, $v_{\mathrm{sh}}$ is the shock speed at which the cosmic-ray population drifts; $\gamma_{\mathrm{CR}}$ is the cosmic-ray Lorentz factor in the population rest frame. $\gamma_{\max } / \Omega_{i}$ is the ratio of the predicted growth rate of the most unstable mode (with wavelength $\lambda_{\max }$ ) to the nonrelativistic ion gyrofrequency. The peak measured perpendicular magnetic field and growth rate are given by $B_{\perp}$ and $\gamma$ in units of their respective reference quantities. Parameters common to all runs include an ion-to-electron mass ratio $m_{i} / m_{e}=50$, a density ratio of ions to cosmic rays $N_{i} / N_{\mathrm{CR}}=50$, and an initial homogeneous magnetic field antiparallel to the cosmic-ray drift such that the Alfvén speed is $v_{A}=0.01 c$.

To minimize statistical noise associated with the particles, we employ a combination of large per-cell particle counts and lowpass filtering of the electric currents arising from their motion. We initialize the simulations with either 20 or 25 plasma ions per cell, and a density ratio of $N_{i} / N_{\mathrm{CR}}=50$ plasma ions per cosmic-ray ion. However, in most runs, we split each cosmic ray into 10 simulated particles that preserve the charge-to-mass ratio but provide better statistics, and the contribution of each particle to currents and densities is weighted accordingly.

Electrons, less massive than the ions by a factor of $m_{i} / m_{e}=$ 50 in all tests, are physically one population but simulated as two populations. In a simulation for which $N_{i}=25$, each cell contains 25 "full" electrons as well as five simulated particles, which add up to half of a split electron. The two simulated electron populations are initialized according to the same distribution function, and behavioral comparisons of the full electrons and the split electrons in the simulations reveal no differences between the two populations, to within statistical limits.

The initial temperature of the plasma is set artificially high, with $v_{e, \text { th }}=0.01 c$, in order to mitigate Buneman-type electrostatic effects arising from the drift between the stationary population of ions and the slowly drifting electrons. Our simulations in N08 demonstrated that cooler plasmas were heated through such effects on a much shorter timescale than any turbulent magnetic-field amplification, but the resulting anisotropy of the ion distribution function in particular persisted on such timescales. A higher initial temperature leads to better preservation of isotropy against the intra-plasma drift. Additionally, using a density ratio $N_{i} / N_{\mathrm{CR}}$ of 50 instead of 3 (the value in N08) significantly reduces $v_{d}$.

The cosmic rays are initialized to be mono-energetic and isotropic in the shock rest frame. This frame drifts in the $-x$ direction, antiparallel to the homogeneous magnetic field, at a speed $v_{\mathrm{sh}}=0.4 c$ or $0.3 c$. We set the magnetic field strength such that the Alfvén speed $v_{A}=0.01 c$, or equivalently that the plasma frequency exceeds the Larmor frequency by a factor of $\omega_{p e} / \Omega_{e} \approx 14.14$.

\subsection{Run-specific parameters}

In Table 1, we summarize the initial conditions and some observations from each of the simulations we discuss. The default parameters are described below, followed by a list of each secondary run's purpose and any deviations from those parameters.

Run A is our large-grid run for this paper, and is the basis for many subsequent "test" simulations. The cosmic rays are isotropic and mono-energetic with Lorentz factor $\gamma_{\mathrm{CR}}=50 \mathrm{in}$ the CR rest frame, which drifts at $v_{\mathrm{sh}}=0.4 c$ in the $-x$-direction in the simulation frame. There are initially 25 plasma ions per cell, and each cosmic-ray ion is split into ten equal parts, for a net of five simulated particles per cell. For these parameters, $\lambda_{\max }=444 \Delta$. In order to capture the nonlinear stage when the dominant structure size exceeds this, we simulate on a grid of $9000 \Delta \times 2400 \Delta$, corresponding to $20.26 \lambda_{\max } \times 5.4 \lambda_{\max }$.

Run $\mathrm{A}^{\prime}$ uses the same physical parameters as Run A, but on a smaller grid, fitting $6.75 \lambda_{\max } \times 3.6 \lambda_{\max }$.

Run A uses a large grid to ameliorate the limitations imposed by periodic boundary conditions, which is particularly relevant during the later evolution when structures in the magnetic field begin to grow quite large. However, for the purpose of analyzing the properties of the upstream medium during the growth and saturation, we simulate a smaller region with a greater detail level. Run B is our test with increased spatial resolution, in which the electron skin length has been increased to $\lambda_{s e}=6 \Delta$, and the particle density reduced to $N_{i}=20$, while holding the other parameters fixed. The grid is $6.75 \lambda_{\max } \times 2.7 \lambda_{\max }$.

Runs $\mathrm{C}$ through $\mathrm{F}$ are tests with varying cosmic-ray Lorentz factors. All use the intermediate value for electron skin length of $\lambda_{s e}=5 \Delta$.

Run $\mathrm{C}$ has a reduced shock speed $v_{\mathrm{sh}}=0.3 \mathrm{c}$. Its cosmic rays also have a reduced Lorentz factor, $\gamma_{\mathrm{CR}}=10$. It is unique among our runs in that it does not split the cosmic rays. The grid for Run $C$ admits $5.4 \lambda_{\max } \times 2.03 \lambda_{\max }$. Run $\mathrm{C}^{\prime}$ is identical to Run $\mathrm{C}$ but splits the cosmic rays.

Runs D, E, and F use the usual shock speed $v_{\mathrm{sh}}=0.4 c$ and cosmic-ray Lorentz factor $\gamma_{\mathrm{CR}}=10,25$, and 200, respectively. Their grid measures $5.76 \lambda_{\max } \times 2.16 \lambda_{\max }$.

\section{RESULTS AND DISCUSSION}

The spatially averaged amplitude of the turbulent components of the magnetic field, parallel and perpendicular to the initial homogeneous field $B_{\| 0}$, are plotted as a function of time in Figure 1 for Run B, representing well the evolution in all runs. The unit of choice for time is the inverse predicted growth rate $\gamma_{\max }^{-1}$. When $t \gamma_{\max } \approx 5$, the field enters a period of exponential 


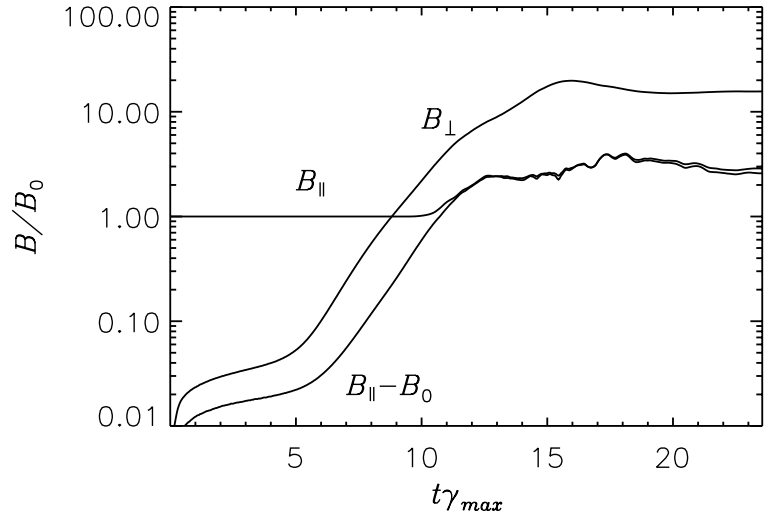

Figure 1. Evolution of the magnetic field components in Run B displaying the growth and saturation of the instability predicted in B04. The magnetic field amplitude is in units of the initial homogeneous field, and time is in dimensionless units set by the calculated inverse growth rate $\gamma_{\max }^{-1}$ from that instability.

growth. As seen in Table 1, the peak growth rate observed is often 10\%-20\% less than $\gamma_{\max }$; this small discrepancy may be primarily a thermal effect. As discussed in N08, the finite temperature of the plasma tends to reduce the maximum growth rate from its value in the cold-plasma limit.

Initially, the growth of the magnetic field is stationary, in the form of non-propagating waves with wave vectors oriented parallel to the cosmic-ray drift and similar in magnitude to $k_{\| \max }$ (Figure 2). The cosmic-ray Larmor radius $r_{\mathrm{CRg}} \gg \lambda_{\max }$, so this is not the resonant interaction of cosmic rays with self-generated Alfvén waves, but is instead the non-resonant generation of an approximately purely growing wave mode. However, density fluctuations begin to appear while $\delta B \ll B_{\| 0}$. The root-mean-square magnitude of the normalized density fluctuations $\left\langle\delta N_{i} / N_{i}\right\rangle$ during the linear growth stage is smaller than $\left\langle\delta B / B_{\| 0}\right\rangle$, typically at a level of 20 to $50 \%$ between $t \gamma_{\max }=5$ and $t \gamma_{\max }=10$. As the turbulent magnetic field becomes comparable in strength to the homogeneous field, the plasma begins to move and the cosmic rays slow down, reducing the current and saturating the magnetic-field amplification at a level of $10-20 B_{\| 0}$.

Any density and velocity fluctuations that arise may contribute to further deviation from a purely growing parallel mode, and may change the environment enough that further field amplification is not favored. We observe that when $B_{\perp} \sim B_{\| 0}$, the growth rate is only gently decreasing and the field still has approximately a parallel-mode structure, but when $B_{\perp} \sim 10 B_{\| 0}$, the spatial organization of the field no longer resembles a parallel mode. As seen in Figure 3, the dominant length scale of the fluctuations grows and significantly exceeds $\lambda_{\max }$. Substantial variation appears along the direction transverse to the drift, reaching the maximum length scale allowed by the periodic boundary conditions of even our largest computational grid. The field growth comes to a stop, saturating generally between 10 and 20 times the amplitude of the initial homogeneous field.

The reduction of the field growth rate accompanies the onset of turbulent plasma motion. Parcels of ambient plasma whose size is comparable to $\lambda_{\max }$ move and collide, giving rise to considerable density fluctuations. Figure 4 (left) indicates that the plasma parcels can attain a considerable range of speeds in the directions transverse to the cosmic-ray drift. The strong perpendicular field and turbulent plasma allow bulk momentum transfer from the cosmic rays to the ambient plasma, so superimposed on the turbulent motions, the plasma begins to drift more and more rapidly in the direction of the cosmic rays, while the cosmic rays simultaneously slow down (Figure 4, right), consistent with the quasi-linear predictions of Winske and Leroy (1984) for non-resonant modes. In our simulations, the speeds converge such that the relative drift is roughly an order of magnitude smaller than its initial value. Riquelme and Spitkovsky (2009) suggest that convergence to a relative velocity approximating the Alfvén speed is sufficient to inhibit further magnetic-field amplification and thus that saturation may take place. Although defining the Alfvén speed for a turbulent magnetic field is complicated, we find that by considering only the projection of the magnetic field onto the drift direction along with the local plasma density averaged over regions of a few $\lambda_{s e}^{2}$ in area, we do observe a "mean" Alfvén speed parallel to the cosmic-ray drift that differs from the post-saturation velocity separation only by a factor of order unity.

In addition to slowing down in bulk, the instantaneous restframe distribution of cosmic rays is modified, as shown in Figure 5. Some anisotropy is introduced, which may have consequences for the modeling both of radiation and particle acceleration. The anisotropy appears in all simulations in a qualitatively similar manner, though the rate at which it develops (as well as the extent to which the CR bulk speed changes) decreases with increasing $\gamma_{\mathrm{CR}}$. The fastest development of restframe anisotropy occurs for cosmic rays whose trajectory is transverse to the drift direction in the simulation frame. During the linear stage of magnetic-field growth, the stationary parallelwave mode with wavelength $\lambda_{\max }$ will be favorable for resonant scattering of cosmic rays whose pitch angle $\cos \mu$ is given by $\lambda_{\max } \Omega_{\mathrm{CR}} / 2 \pi=v_{\|}=\mu c\left(1-\gamma_{\mathrm{CR}}^{-2}\right)^{1 / 2}$. For the parameters in our simulations and in agreement with the work of Luo and Melrose (2009), $\mu$ is of order $10^{-2}$, corresponding to those cosmic rays in which the aforementioned anisotropy is first seen. Additional scattering and acceleration of cosmic rays may occur in the electric fields associated with magnetic fields being transported in the turbulent flow during the nonlinear stage. Second-order Fermi acceleration is possible in such a turbulent environment. The electric fields we observe during saturation are typically an order of magnitude smaller than the magnetic fields. A Lorentz transformation into the global plasma rest frame reduces the electric fields only from about $t \gamma_{\max }=15$ onward, in agreement with the behavior of the plasma bulk speed. If we transform the EM fields into the local plasma rest frame, thereby accounting for its turbulent motion, we see a reduction of the electric fields beginning at $t \gamma_{\max } \approx 10$. After about $t \gamma_{\max }=15$, less than $20 \%$ of the original electric field remains, demonstrating that the dominant fraction of the electric field in our simulation arises from the transport of magnetic fields. This indicates that pure elastic scattering may be a poor assumption, if one considers the diffusive transport of high-energy cosmic rays in the upstream region of young SNR. Runs C-F with varying cosmic-ray Lorentz factors illustrated principally that "stiffer" cosmic rays took slightly longer to begin slowing down and were marginally more resistant to the onset of rest-frame anisotropy, but there was no qualitative difference in the linear growth of the magnetic field nor in the saturation mechanism.

The interactions between the shock itself and any spatial variations not only in plasma density but also in velocity are known to introduce vorticity in the downstream region that may amplify the magnetic field (Giacalone and Jokipii 2007; Inoue et al. 2009). They may also have a non-negligible impact on the injection process or the scattering properties of the downstream medium, and consequently the resulting distribution of energetic 


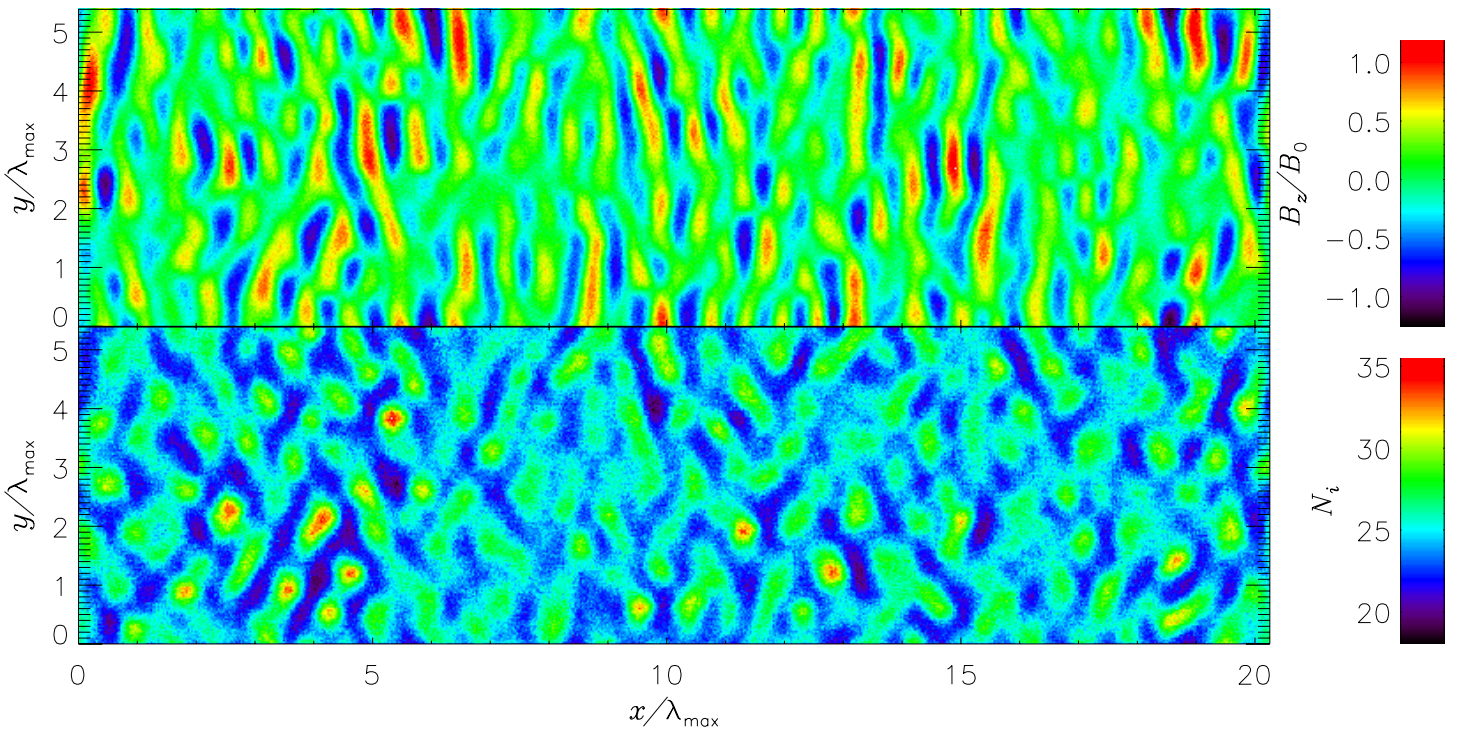

Figure 2. Perpendicular magnetic field in units of $B_{\| 0}$ (top) and density of plasma ions (bottom) at $t \gamma_{\max }=7.0$ for Run $\mathrm{A}$, when $\left\langle\delta B / B_{\| 0}\right\rangle \approx 0.50$. The parallel-mode structure is clearly visible in the magnetic field. Localized density fluctuations about the mean (25 ions per cell) are smaller, with an amplitude of $\left\langle\delta N_{i} / N_{i}\right\rangle \approx 0.08$. (A color version of this figure is available in the online journal.)

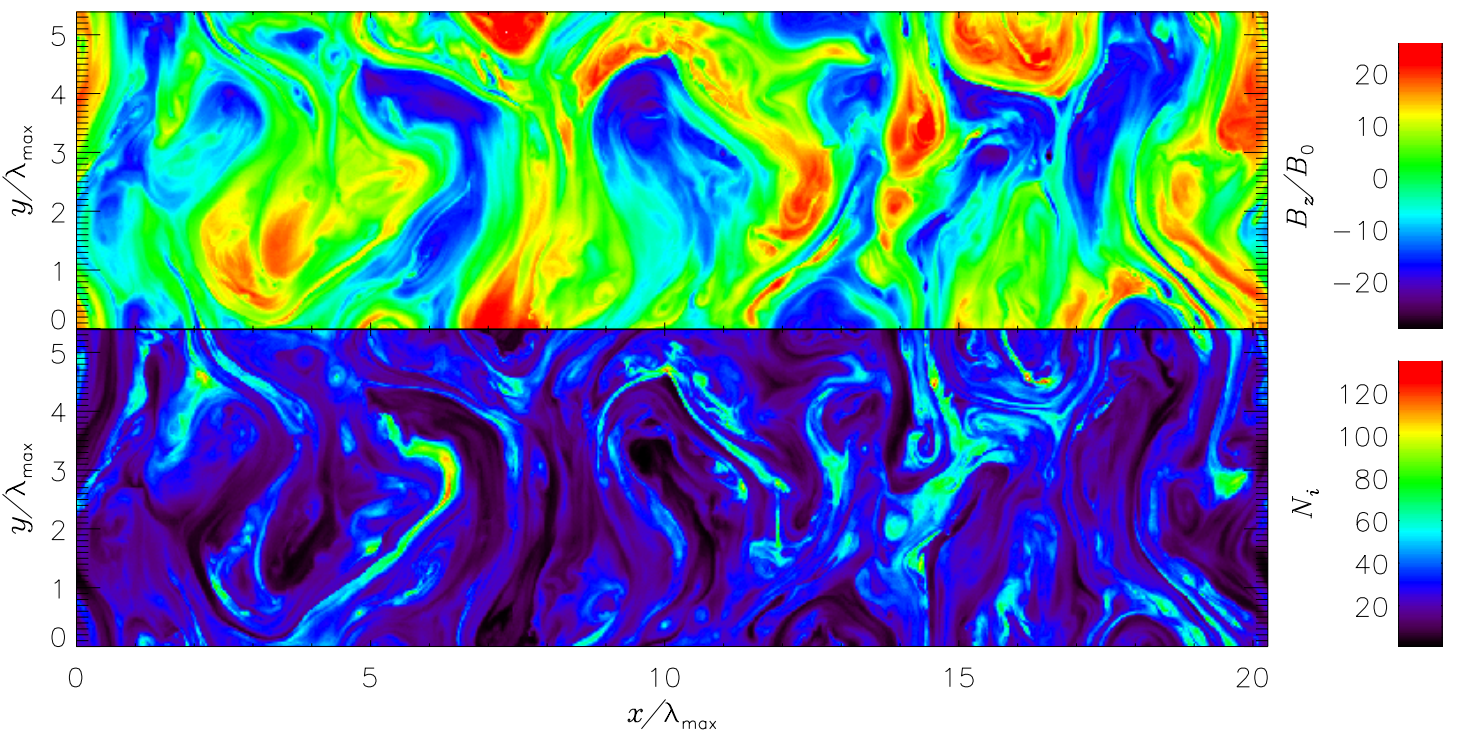

Figure 3. As in Figure 2, the perpendicular magnetic field and density of plasma at $t \gamma_{\max }=14.0$ in Run A. The plasma density fluctuations are strong and well correlated with the magnetic field structures during the nonlinear stage of magnetic field growth. The structures move rapidly on oblique trajectories at this time in the simulation (see Figure 4).

(A color version of this figure is available in the online journal.)
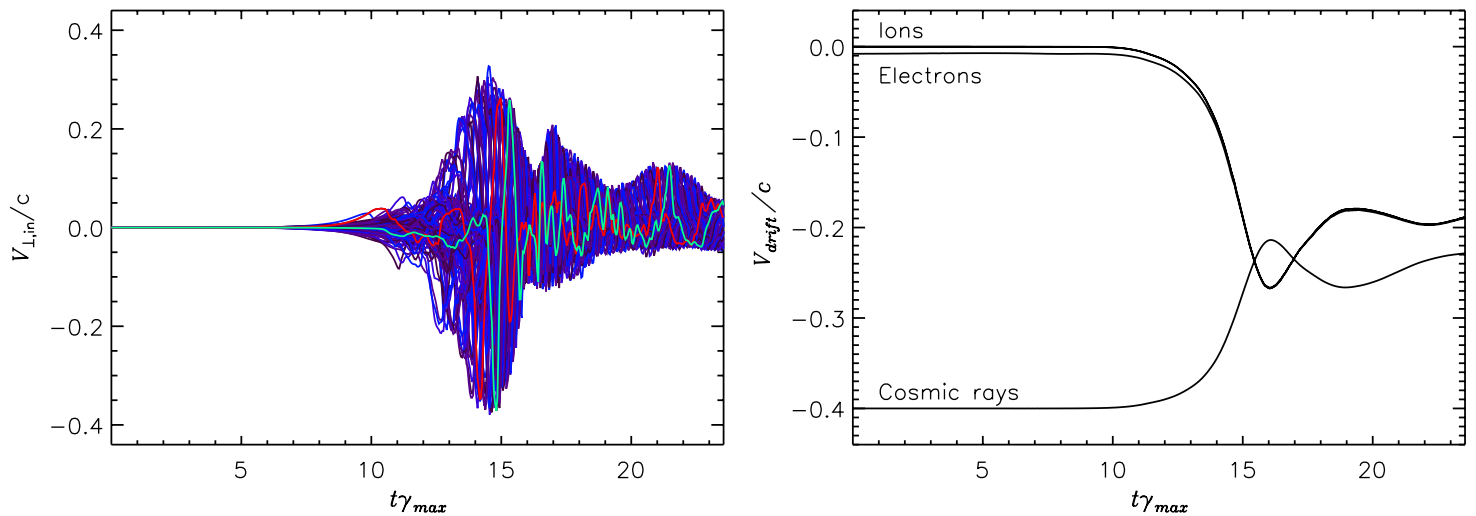

Figure 4. Left: from Run B, the transverse in-plane velocity of the plasma in regions of size $\left(0.45 \lambda_{\max }\right)^{2}$. Two regions have been selected and superimposed in lighter colors for clarity. Right: in the same run, momentum transfer from the cosmic rays to the background plasma removes their relative drift, limiting the amplification of the magnetic field. The "overshoot" at $t \gamma_{\max } \sim 16$ appears in the small-grid runs but is not present in Run A, with the larger grid, so it is likely a consequence of the periodic boundary conditions.

(A color version of this figure is available in the online journal.) 


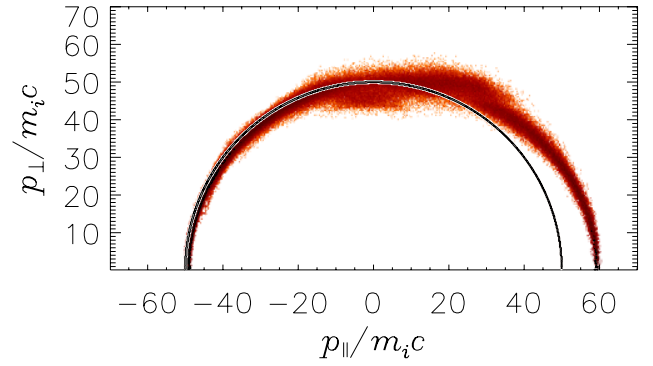

Figure 5. Phase-space distribution of cosmic rays in the instantaneous rest frame of the population at time $t \gamma_{\max } \approx 15.7$ in Run B, compared with the initial distribution (thin semicircle). In addition to revealing the anisotropy in the forms of stretching and broadening that began during the linear magneticfield growth, the instantaneous rest frame differs from the initial frame by approximately $0.18 c$.

(A color version of this figure is available in the online journal.)

particles (Malkov and Drury 2001). If the injection mechanism depends primarily on highly localized processes, as opposed to being a product of the average conditions over large spatiotemporal scales, then the presence of appropriately distributed inhomogeneities might reduce the availability of readily accelerated particles in one region, with no guarantee of a corresponding increase in a neighboring region. Particularly relevant if injection is ultimately more efficient at a parallel shock than at a perpendicular shock, the development of a turbulent magnetic field in the formerly parallel region via this streaming instability would reduce the overall quasi-parallel fraction of the shock's surface if comparable turbulence were not established in the initially quasi-perpendicular regions. Beyond injection, the cosmic-ray transport properties upstream and downstream contribute significantly to the acceleration efficiency and the maximum energy cosmic rays can attain. Marcowith et al. (2006) investigated the sensitivity of the cosmic-ray diffusion coefficient to the character of the turbulence, and found that, in particular, anisotropic turbulence may lead to somewhat higher maximum particle energy.

In addition to possible implications for the spectrum of accelerated particles, the character of the upstream magnetic turbulence also eventually contributes to the emission properties of the downstream medium. The presence of anisotropy in the turbulence after being shock-compressed may lead to a larger degree of polarization in the radio synchrotron emission than might otherwise be expected (Stroman and Pohl 2009).

The turbulent motion in the upstream medium may be significant for self-consistent models of cosmic-ray acceleration. In our simulations, plasma parcels attain velocities that exceed the initial Alfvén speed and collide, producing "fronts" whose effects may include further localized field amplification or, perhaps quite importantly, compression and heating of the upstream medium. We observe that heating of the ambient plasma occurs cospatially with the interfaces between rapidly moving plasma parcels and the slower regions into which they are pressing. In Run $\mathrm{B}$, for instance, the average kinetic energy per ion is of order $10^{-6} m_{i} c^{2}$ at $t \gamma_{\max }=3.8$, before the linear growth has begun, and is slightly higher but still of that order at $t \gamma_{\max }=7.6$, during linear growth. But at $t \gamma_{\max }=11.5$, the beginning of saturation, the mean kinetic energy per ion has increased to $\sim 5 \times 10^{-4} \mathrm{~m}_{i} \mathrm{c}^{2}$, and at $t \gamma_{\max }=15.3$, it has climbed to $10^{-2} m_{i} c^{2}$. The common and convenient assumption that the entire amplification and saturation process proceeds in a low-temperature regime must be carefully considered if conditions allow for turbulence to de- velop rapidly in the precursor, but as Vladimirov et al. (2008) observe, the substantial heating expected from the dissipation of even a small fraction of upstream turbulence may significantly increase the rate at which particles are injected into the acceleration process.

The reduction of the cosmic-ray current (by the converging drift velocities of their population and the ambient plasma) serves to underscore the need to treat these particles kinetically. The back-reaction from the turbulent plasma and amplified magnetic field is non-negligible, with the most prominent consequence being saturation of the field growth at a lower amplitude than might be expected from a constant cosmic-ray current (Ohira et al. 2009).

Finally, it is worth noting that although the initial dominant mode of magnetic-field amplification agrees with the calculations of B04, the later evolution and saturation mechanism have much in common with the simulations performed in N08, in which the choice of simulation parameters exposed an oblique filamentary mode as the dominant instability. It seems it doesn't matter what form the initial linear instability takes.

An important consideration in interpreting our findings is that the ability of our simulations to portray with accuracy the evolution of the magnetic field and the plasma properties at very late times, beyond the saturation, is limited. In addition to the limitation imposed by the finite size of the simulation domain, the assumptions of environmental homogeneity become increasingly unsound. Changes in the plasma flow speed imply density adjustments in order to conserve mass flux (Ohira et al. 2009). Also, as the shock approaches, spatial gradients in the cosmic-ray precursor and ambient plasma properties play an increasingly important role. Furthermore, the time in which upstream amplification can occur before the system is overtaken by the shock is only $\sim 40 \gamma_{\max }^{-1}$ for our parameters, assuming efficient Bohm-type diffusion so that the shock sweep-up timescale is $c r_{\mathrm{CRg}} / 3 v_{\mathrm{sh}}^{2}$ (N08). Thus, field growth is inevitably limited even in the absence of saturation.

It bears mentioning that to a first approximation, the effects of a changing environment will be limited. As mentioned in Section 2.1, the parameters of the streaming instability are agnostic to the details of the cosmic-ray distribution. Thus, although an approaching precursor implies a continual softening of the local cosmic-ray energy spectrum due to the arrival of particles over an increasingly inclusive energy range, it is only the increased overall flux that matters. Until surplus high-energy electrons are included, the net cosmic-ray current will increase, whose effect according to Equation 1 will be to amplify the parallel mode described therein on shorter time- and lengthscales, potentially well separated from the large scales attained by the turbulence generated earlier. Even this effect is difficult to predict with confidence: not only will cosmic rays that arrive during saturation encounter a turbulent upstream medium, but also may themselves have been influenced by prior unstable evolution nearer to the shock. In any case, since the precursor length scale is proportional to the diffusion coefficient, and hence to the energy for Bohm diffusion, cosmic-ray particles of significantly lower energy (and higher flux) are brought in only shortly prior to capture by the shock.

Although the non-resonant streaming instability is concerned only with the current the cosmic rays carry (and, as demonstrated by Pelletier et al. 2006 and Luo and Melrose 2009, is largely produced by the return current in the plasma), this or other instabilities thus encountered may introduce anisotropy and inhomogeneity into the local cosmic-ray distribution, with potentially 
non-trivial consequences. As increased computational capacity and improved models become available, we can expect simulations representing a significant extent of the precursor to yield valuable insight into these interrelated and nonlinear processes occurring there.

\section{SUMMARY AND CONCLUSIONS}

We have simulated the turbulent amplification of the interstellar magnetic field upstream of a nonrelativistic shock using a kinetic 2.5-dimensional particle-in-cell code.

We observe the following:

1. That the non-resonant streaming instability (B04) is seen initially for our choice of parameters, but with fluctuations appearing in the plasma density.

2. That the plasma quickly evolves and saps the bulk momentum from the drifting cosmic rays.

3. The reduction of relative drift effectively removes the cosmic-ray current and saturates the magnetic-field amplification to $\sim 20 B_{\| 0}$.

4. This saturation mechanism is independent of the initial linear instability, occurring as a result of the back-reaction on the cosmic rays.

5. Strong transverse plasma motions arise in conjunction with the turbulent magnetic field exceeding the homogeneous background field.

6. Interactions between differently-moving plasma parcels give rise to significant fluctuations in density and temperature of the upstream interstellar medium.

7. The plasma begins to drift, suggestive of a cosmic raymodified shock, until its speed approximately matches that of the cosmic-ray population.

8. The phase-space distribution of cosmic rays undergoes significant changes: the development of anisotropy within the population rest frame beginning during the linear growth of the magnetic field, and decrease in bulk speed associated temporally with the saturation of the magnetic field.

9. Evolution past this point is beyond the reach of our current simulation method, as it requires additional input to reflect the changing external environment.

The growth of non-resonant streaming instabilities in the cosmic-ray precursor of supernova shocks may contribute to significant turbulent amplification of the magnetic field upstream of the shock. However, the amplitudes achieved via any one process are likely to be somewhat less than suggested in the artificial case of constant cosmic-ray current. Simulations that incorporate incrementally more of the environmental changes occuring in a real precursor scenario can be expected as computational capacity increases, although it may take innovations beyond the foreseeable future to make a realistic particle-incell approach tractable for such a simulation (Vladimirov et al. 2008).

This research was supported in part by the National Science Foundation both through TeraGrid resources provided by NCSA (Catlett et al. 2007) and under grant No. PHY05-51164. The work of J.N. is supported by MNiSW research project $\mathrm{N}$ N203 393034, and The Foundation for Polish Science through the HOMING program, which is supported by a grant from Iceland, Liechtenstein, and Norway through the EEA Financial Mechanism.

\section{REFERENCES}

Bell, A. R. 2004, MNRAS, 353, 550

Buneman, O. 1993, Computer Space Plasma Physics: Simulation Techniques and Software, ed. H. Matsumoto \& Y. Omura - (Tokyo: Terra), 67

Catlett, C., et al. 2007, TeraGrid: Analysis of Organization, System Architecture, and Middleware Enabling New Types of Applications (Amsterdam: IOS Press)

Giacalone, J., \& Jokipii, J. R. 2007, ApJ, 663, L41

Greenwood, A. D., Cartwright, K. L., Luginsland, J. W., \& Baca, E. A. 2004, J Comput. Phys., 201, 665

Inoue, T., Yamazaki, R., \& Inutsuka, S.-i. 2009, ApJ, 695, 825

Luo, Q., \& Melrose, D. 2009, MNRAS, 397, 1402

Malkov, M. A., \& Drury, L. O. 2001, Rep. Prog. Phys., 64, 429

Marcowith, A., Lemoine, M., \& Pelletier, G. 2006, A\&A, 453, 193

Niemiec, J., Pohl, M., Stroman, T., \& Nishikawa, K. I. 2008, ApJ, 684, 1174

Ohira, Y., Reville, B., Kirk, J. G., \& Takahara, F. 2009, ApJ, 698, 445

Pelletier, G., Lemoine, M., \& Marcowith, A. 2006, A\&A, 453, 181

Reville, B., Kirk, J. G., \& Duffy, P. 2009, ApJ, 694, 951

Reynolds, S. P. 2008, ARA\&A, 46, 89

Riquelme, M. A., \& Spitkovsky, A. 2009, ApJ, 694, 626

Stroman, W., \& Pohl, M. 2009, ApJ, 696, 1864

Umeda, T., Omura, Y., Tominaga, T., \& Matsumoto, H. 2003, Comput. Phys. Commun., 156, 73

Vladimirov, A. E., Bykov, A. M., \& Ellison, D. C. 2008, ApJ, 688, 1084

Winske, D., \& Leroy, M. M. 1984, J. Geophys. Res., 89, 2673

Zirakashvili, V. N., Ptuskin, V. S., \& Völk, H. J. 2008, ApJ, 678, 255 